\title{
ON SUMS AND PRODUCTS OF UNBOUNDED OPERATORS IN HILBERT SPACE
}

\author{
BY \\ M. J. J. LENNON
}

\begin{abstract}
The characteristic matrices (in the sense of Stone) of the sum and product of two closed linear operators in Hilbert space are found in terms of the characteristic matrix of each operator. From these, necessary and sufficient conditions for the domain of the sum or product to be dense are found, and a new simple condition for the density of the domain of the sum is proved. The ideas developed are applied to the direct integral decomposition of closed linear operators.
\end{abstract}

In this paper, we are concerned with the problems of adding and multiplying partially defined linear operators on a Hilbert space. Let $A$ and $B$ be linear operators on a Hilbert space $\mathscr{H}$, with domains $\mathscr{D}(A)$ and $\mathscr{D}(B)$, and graphs $\mathcal{G}(A)$ and $\mathcal{G}(B)$ in $\mathcal{I C} \oplus \mathscr{H}$. We say $A$ is closable if $\overline{\mathcal{G}(A)}$ is the graph of a linear operator. Then it is well known that $\mathscr{D}(A+B)=\mathscr{D}(A) \cap \mathscr{D}(B)$ may be $\{0\}$, even though $\mathscr{D}(A)$ and $\mathscr{D}(B)$ are dense. Also $A+B$ may not be closable, even though $A$ and $B$ are closed (i.e. have closed graphs).

We consider the problem of finding general conditions which enable one to determine when these undesirable cases do not arise. Our approach is by way of the "characteristic matrix" of Stone [4], and we find the characteristic matrix of the sum $\frac{1}{2}(A+B)$ and product $B A$ of two general closed linear operators $A$ and $B$. Our result is in the form of the limit of an expression in the characteristic matrix elements in $A$ and $B$, but we also obtain a simple formula in these matrix elements, which determines when $\mathscr{D}(A+B)$ is dense.

In the final section we consider one application of these results, to the direct integral theory of closed linear operators, as developed by Nussbaum [2]. (It may be appropriate to point out here that some of his results were stated earlier by Pallu de Barrière [3].) We obtain two theorems, of the form "the sum and product of the integrals is the integral of the sums and products", which seem to be the most general that can be obtained in this context.

1. Characteristic matrices. Let $\mathcal{H}$ be a fixed complex Hilbert space; we use the notation:

$$
\mathcal{K}^{2}=\mathcal{H} \oplus \mathcal{H}, \quad \mathcal{K}^{3}=\mathcal{H} \oplus \mathcal{H} \oplus \mathcal{K}
$$

The operator 1 is the identity operator; the Hilbert space on which it acts may

Received by the editors January 30, 1973 and, in revised form, May 25, 1973.

AMS (MOS) subject classifications (1970). Primary 47D99, 28A45, 46G99.

Key words and phrases. Unbounded linear operators, characteristic matrix, direct integral of operators. 
be deduced from the context. If $A$ is a closable linear operator in $\mathscr{T}$, the closure of $A$ is denoted $[A]^{-}$.

Let $A$ be a linear operator in $\mathcal{H}$, domain $\mathscr{Q}(A)$ and range $\mathscr{R}(A)$ in $\mathcal{H}$, and graph $\mathcal{S}(A)$ in $\mathcal{T}^{2}$. Let $G(A)$ be the orthogonal projection onto the closure of $\mathcal{S}(A)$. We may write $G(A)$ as a matrix of bounded linear operators on $\mathcal{H}$,

$$
G(A)=\left[\begin{array}{ll}
A_{11} & A_{12} \\
A_{21} & A_{22}
\end{array}\right] .
$$

This matrix is called the characteristic matrix of $A$. We have the following theorem.

Theorem 1.1. A $2 \times 2$ matrix $\left(A_{i j}\right)$ of bounded linear operators on $\mathcal{H C}$ is the characteristic matrix of a closed linear operator on $\mathcal{H}$ if and only if

(i) $\sum_{j=1,2} A_{i j} A_{j k}=A_{i k}, i, k=1,2$,

(ii) $A_{i j}^{*}=A_{j i}, i, j=1,2$,

(iii) $1-A_{22}$ is injective.

Furthermore, if $\left(A_{i j}\right)$ is the characteristic matrix of the closed operator $A$, we have

(iv) $0 \leq A_{11} \leq 1,0 \leq A_{22}<1,\left\|A_{12}\right\| \leq 1$,

(v) $\mathscr{D}(A)=\left\{A_{11} x_{1}+A_{12} x_{2} \mid x_{1}, x_{2} \in \mathcal{H C}\right\}, A: A_{11} x_{1}+A_{12} x_{2} \rightarrow A_{21} x_{1}+A_{22} x_{2}$.

Proof. See Stone [4], and Nussbaum [2].

If $A$ and $B$ are any two closed linear operators in 3 , we shall use the notation $A_{i j}, B_{i j}$, for the elements of their characteristic matrices throughout the rest of the paper.

The usefulness of the concept of characteristic matrix is increased by the fact that it is possible to calculate the operators $A_{i j}$ explicitly if $\mathscr{D}(A)$ is dense. In the following theorem we summarise information which is obtainable from the operators $A_{i j}$.

Theorem 1.2. Let $A$ be a closed linear operator on $\mathcal{X}$ and $\left(A_{i j}\right)$ its characteristic matrix. Then we have:

(i) $\mathcal{D}(A)$ is dense iff $A_{11}$ is injective. In this case,

$$
\begin{array}{ll}
A_{11}=\left(1+A^{*} A\right)^{-1}, & A_{12}=A^{*}\left(1+A A^{*}\right)^{-1}, \\
A_{21}=A\left(1+A^{*} A\right)^{-1}, & A_{22}=A A^{*}\left(1+A A^{*}\right)^{-1} .
\end{array}
$$

We also have

$$
G\left(A^{*}\right)=\left[\begin{array}{cc}
1-A_{22} & A_{21} \\
A_{12} & 1-A_{11}
\end{array}\right] .
$$

(ii) $A$ is injective iff $1-A_{11}$ is injective. In this case,

$$
G\left(A^{-1}\right)=\left[\begin{array}{ll}
A_{22} & A_{21} \\
A_{12} & A_{11}
\end{array}\right]
$$


(iii) $A$ is bounded iff $A_{11}>a 1$ for some a $>0$.

(iv) $A$ is normal iff all the $A_{i j}$ are normal and commute with each other.

Proof. See Stone [4] and Nussbaum [2].

2. Bicharacteristic matrices. Let $A$ and $B$ be two closed linear operators on $\mathcal{T}$. Definition. The sum bigraph, $\delta(A, B)$, is the subspace of $\mathcal{T C}^{4}$ defined by

$$
\{(x, A x, x, B x) \mid x \in \mathscr{D}(A) \cap \mathscr{D}(B)\} .
$$

The product bigraph, $\mathscr{P}(A, B)$, is the subspace of $\mathcal{K}^{4}$ defined by

$$
\{(x, A x, A x, B A x) \mid x \in \mathscr{D}(A), A x \in \mathscr{D}(B)\} .
$$

Lemma 2.1. $\delta(A, B)$ and $\mathscr{P}(A, B)$ are closed subspaces.

Proof. Let $\left\{\left(x_{n}, A x_{n}, x_{n}, B x_{n}\right)\right\}$ be a Cauchy sequence in $\delta(A, B)$. Then $\left\{x_{n}\right\}$, $\left\{A x_{n}\right\},\left\{B x_{n}\right\}$ are all Cauchy in $\mathscr{K}$, so there exist $x, y, z \in \mathcal{H}$ such that $x_{n} \rightarrow x$, $A x_{n} \rightarrow y, B x_{n} \rightarrow z$. Since $A$ and $B$ are closed, $x \in \mathscr{D}(A) \cap \mathscr{D}(B), y=A x, z$ $=B x$, and hence $\left\{\left(x_{n}, A x_{n}, x_{n}, B x_{n}\right)\right\}$ converges to an element of $\delta(A, B)$.

The proof for $\mathcal{P}(A, B)$ is similar.

Let $S(A, B)$ and $P(A, B)$ be the orthogonal projections onto $S(A, B)$ and $\mathscr{P}(A, B)$ respectively. We may write these projections as $4 \times 4$ matrices, $\left(S_{i j}\right)$ and $\left(P_{i j}\right)$ respectively, of bounded linear operators on $\mathcal{H}_{6}$. We call these matrices the bicharacteristic matrices of $A$ and $B$.

Proposition 2.2. (i) $\sum_{j=1}^{4} S_{i j} S_{j k}=S_{i k}, \sum_{j=1}^{4} P_{i j} P_{j k}=P_{i k}, i, k=1, \ldots, 4$.

(ii) $S_{i j}^{*}=S_{j i}, P_{i j}^{*}=P_{j i}, i, j=1, \ldots, 4$.

(iii) $\mathscr{D}(A) \cap \mathscr{D}(B)$ is dense iff $S_{11}$ is injective.

(iv) $\{x \in \mathscr{D}(A) \mid A x \in \mathscr{D}(B)\}$ is dense iff $P_{11}$ is injective.

Proof. The proof may be carried out in exactly the same way as the analogous facts are proved in [2] and [4].

3. Calculation of the bicharacteristic matrices. In this section we find an expression for each $S_{i j}$ and $P_{i j}$, in terms of the bounded operators $A_{i j}$ and $B_{i j}$.

Let $E_{1}$ be the orthogonal projection onto the closed subspace of $\mathcal{F}^{4}$,

$$
\left\{\left(x_{1}, x_{2}, x_{3}, x_{4}\right) \mid x_{1}=x_{3}\right\},
$$

and let $E_{2}$ be the orthogonal projection onto $S(A) \oplus \mathcal{S}(B)$. Then it is easy to see that $\delta(A, B)$ is the intersection of $E_{1} \mathcal{H}^{4}$ and $E_{2} \mathcal{F}^{4}$. It follows [5, Theorem 13.7] that $S(A, B)$ is the limit in the strong operator topology of the sequence $\left\{E_{1}\left(E_{2} E_{1}\right)^{n}\right\}$. Since

$$
E_{1}=\left[\begin{array}{cccc}
\frac{1}{2} 1 & 0 & \frac{1}{2} 1 & 0 \\
0 & 1 & 0 & 0 \\
\frac{1}{2} 1 & 0 & \frac{1}{2} 1 & 0 \\
0 & 0 & 0 & 1
\end{array}\right], \quad E_{2}=\left[\begin{array}{cccc}
A_{11} & A_{12} & 0 & 0 \\
A_{21} & A_{22} & 0 & 0 \\
0 & 0 & B_{11} & B_{12} \\
0 & 0 & B_{21} & B_{22}
\end{array}\right],
$$


$S(A, B)$ may be calculated explicitly. The result would not be of much use, however, if it were not that great simplification is possible. We give the final result of this simplification and prove its correctness by induction.

Theorem 3.1. Let $A$ and $B$ be any two closed linear operators, and let $\left(A_{i j}\right),\left(B_{i j}\right)$ be their characteristic matrices. For $n=1,2, \ldots$, define

$$
S_{n}=-\frac{1}{2} \sum_{i=1}^{n}\left(\begin{array}{l}
n \\
i
\end{array}\right)\left[-\frac{1}{2}\left(A_{11}+B_{11}\right)\right]^{i-1},
$$

and let $X$ be any one of $A_{11}-B_{11}, A_{21}, B_{21}$, and $Y$ any one of $A_{11}-B_{11}, A_{12}, B_{12}$. Then the limit in the strong operator topology of the sequence $\left\{X S_{n} Y\right\}$ exists. Denoting this limit by $[X S Y]$ we have

$$
\begin{aligned}
& S_{11}=S_{13}=S_{31}=S_{33}=\frac{1}{4}\left(A_{11}+B_{11}\right)+\frac{1}{4}\left[\left(A_{11}-B_{11}\right) S\left(A_{11}-B_{11}\right)\right] \\
& S_{12}=S_{32}=1 / 2 A_{12}+1 / 2\left[\left(A_{11}-B_{11}\right) S A_{12}\right], \\
& S_{21}=S_{23}=1 / 2 A_{21}+1 / 2\left[A_{21} S\left(A_{11}-B_{11}\right)\right], \\
& S_{14}=S_{34}=1 / 2 B_{12}-1 / 2\left[\left(A_{11}-B_{11}\right) S B_{12}\right], \\
& S_{41}=S_{43}=1 / 2 B_{21}-1 / 2\left[B_{21} S\left(A_{11}-B_{11}\right)\right], \\
& S_{24}=-\left[A_{21} S B_{12}\right], \\
& S_{42}=-\left[B_{21} S A_{12}\right], \\
& S_{22}=A_{22}+\left[A_{21} S A_{12}\right], \\
& S_{44}=B_{22}+\left[B_{21} S B_{12}\right] .
\end{aligned}
$$

Proof. First define $S_{0}=0$, and note that

$$
S_{n}\left(1-\frac{1}{2}\left(A_{11}+B_{11}\right)\right)=S_{n+1}+\frac{1}{2} 1, \quad n=0,1,2, \ldots
$$

Let $C$ be the operator $A_{11}-B_{11}$. We shall first prove by induction

$$
\begin{aligned}
E_{1}\left(E_{2} E_{1}\right)^{n+1}= & {\left[\begin{array}{cccc}
\frac{1}{4}\left(A_{11}+B_{11}\right) & \frac{1}{2} A_{12} & \frac{1}{4}\left(A_{11}+B_{11}\right) & \frac{1}{2} B_{12} \\
\frac{1}{2} A_{21} & A_{22} & \frac{1}{2} A_{21} & 0 \\
\frac{1}{4}\left(A_{11}+B_{11}\right) & \frac{1}{2} A_{12} & \frac{1}{4}\left(A_{11}+B_{11}\right) & \frac{1}{2} B_{12} \\
\frac{1}{2} B_{21} & 0 & \frac{1}{2} B_{21} & B_{22}
\end{array}\right] } \\
& +\left[\begin{array}{cccc}
\frac{1}{4} C S_{n} C & \frac{1}{2} C S_{n} A_{12} & \frac{1}{4} C S_{n} C & -\frac{1}{2} C S_{n} B_{12} \\
\frac{1}{2} A_{21} S_{n} C & A_{21} S_{n} A_{12} & \frac{1}{2} A_{21} S_{n} C & -A_{21} S_{n} B_{12} \\
\frac{1}{4} C S_{n} C & \frac{1}{2} C S_{n} A_{12} & \frac{1}{4} C S_{n} C & -\frac{1}{2} C S_{n} B_{12} \\
-\frac{1}{2} B_{21} S_{n} C & -B_{21} S_{n} A_{12} & -\frac{1}{2} B_{21} S_{n} C & B_{21} S_{n} B_{12}
\end{array}\right] .
\end{aligned}
$$

This is clearly true for $n=0$. Assume it is true for $n=k$, and let 


$$
\begin{aligned}
\left(R_{i j}\right) & =E_{1}\left(E_{2} E_{1}\right)^{k+2} \\
& =E_{1}\left(E_{2} E_{1}\right)^{k+1}\left[\begin{array}{cccc}
\frac{1}{2} A_{11} & A_{12} & \frac{1}{2} A_{11} & 0 \\
\frac{1}{2} A_{21} & A_{22} & \frac{1}{2} A_{21} & 0 \\
\frac{1}{2} B_{11} & 0 & \frac{1}{2} B_{11} & B_{12} \\
\frac{1}{2} B_{21} & 0 & \frac{1}{2} B_{21} & B_{22}
\end{array}\right] .
\end{aligned}
$$

Then it is easy to check that each $R_{i j}$ has the required form, using the relationship (*) and relationships derived from Theorem 1.1, such as $A_{12} A_{21}$ $=A_{11}-A_{11}^{2}$. For example, we have

$$
\begin{aligned}
R_{11}= & \frac{1}{8}\left(A_{11}+B_{11}\right)^{2}+\frac{1}{8} C S_{k} C\left(A_{11}+B_{11}\right)+\frac{1}{4} A_{12} A_{21} \\
& +\frac{1}{4} B_{12} B_{21}+\frac{1}{4} C S_{k}\left(A_{12} A_{21}-B_{12} B_{21}\right) \\
= & \frac{1}{4}\left(A_{11}+B_{11}\right)+\frac{1}{8}\left(-A_{11}^{2}+A_{11} B_{11}+B_{11} A_{11}-B_{11}^{2}\right) \\
& +\frac{1}{8} C S_{k}\left(A_{11}^{2}-B_{11} A_{11}+A_{11} B_{11}-B_{11}^{2}+2 A_{11}-2 A_{11}^{2}-2 B_{11}+2 B_{11}^{2}\right) \\
= & \frac{1}{4}\left(A_{11}+B_{11}\right)-\frac{1}{8}\left(A_{11}-B_{11}\right)^{2}+\frac{1}{8} C S_{k}\left(2\left(1-\frac{1}{2}\left(A_{11}+B_{11}\right)\right) C\right) \\
= & \frac{1}{4}\left(A_{11}+B_{11}\right)+\frac{1}{4} C S_{k+1} C .
\end{aligned}
$$

Since we know that $\left\{E_{1}\left(E_{2} E_{1}\right)^{n}\right\}$ converges in the strong operator topology to $S(A, B)$, it follows that the operators $X S_{n} Y$ converge in the strong operator topology to bounded operators, where $X$ is any one of $C, A_{21}, B_{21}$ and $Y$ any one of $C, A_{12}, B_{12}$. This completes the proof of the theorem.

The corresponding theorem for the product bicharacteristic matrix can now be deduced by analogy from the observation that $\mathcal{P}(A, B)$ is the intersection of $E_{2} \mathcal{T C}^{4}$ and the subspace $\left\{\left(x_{1}, x_{2} x_{3}, x_{4}\right) \mid x_{2}=x_{3}\right\}$.

Theorem 3.2. Let $A$ and $B$ be any two closed linear operators, and let $\left(A_{i j}\right),\left(B_{i j}\right)$ be their characteristic matrices. For $n=1,2, \ldots$ define

$$
T_{n}=-\frac{1}{2} \sum_{i=1}^{n}\left(\begin{array}{c}
n \\
i
\end{array}\right)\left[-\frac{1}{2}\left(A_{22}+B_{11}\right)\right]^{i-1}
$$

and let $X$ be any one of $A_{22}-B_{11}, A_{12}, B_{21}$, and $Y$ any one of $A_{22}-B_{11}, A_{21}, B_{12}$. Then the limit in the strong operator topology of the sequence $\left\{X T_{n} Y\right\}$ exists. Denoting this limit by $[X T Y]$ we have

$$
\begin{aligned}
& P_{22}=P_{23}=P_{32}=P_{33}=\frac{1}{4}\left(A_{22}+B_{11}\right)+\frac{1}{4}\left[\left(A_{22}-B_{11}\right) T\left(A_{22}-B_{11}\right)\right] \\
& P_{12}=P_{13}=\frac{1}{2} A_{12}+\frac{1}{2}\left[A_{12} T\left(A_{22}-B_{11}\right)\right] \\
& P_{21}=P_{31}=\frac{1}{2} A_{21}+\frac{1}{2}\left[\left(A_{22}-B_{11}\right) T A_{21}\right] \\
& P_{24}=P_{34}=\frac{1}{2} B_{12}-\frac{1}{2}\left[\left(A_{22}-B_{11}\right) T B_{12}\right] \\
& P_{42}=P_{43}=\frac{1}{2} B_{21}-\frac{1}{2}\left[B_{21} T\left(A_{22}-B_{11}\right)\right]
\end{aligned}
$$




$$
\begin{aligned}
& P_{11}=A_{11}+\left[A_{12} T A_{21}\right], \\
& P_{14}=-\left[A_{12} T B_{12}\right], \\
& P_{41}=-\left[B_{21} T A_{21}\right], \\
& P_{44}=B_{22}+\left[B_{21} T B_{12}\right] .
\end{aligned}
$$

Proof. Letting $E_{3}$ be the orthogonal projection onto the closed subspace $\left\{\left(x_{1}, x_{2}, x_{3}, x_{4}\right) \mid x_{2}=x_{3}\right\}$, it is easy to see that the substitution of $E_{3}$ for $E_{1}$ in the proof of Theorem 3.1 is equivalent to the substitutions

$$
A_{11} \rightarrow A_{22}, A_{22} \rightarrow A_{11}, A_{12} \rightarrow A_{21}, A_{21} \rightarrow A_{12},
$$

and the exchange of the first and second rows, and first and second columns, in $E_{1}\left(E_{2} E_{1}\right)^{n+1}$. The present theorem then follows by making these same substitutions in the statement of the preceding theorem.

In many cases we can compute the $S_{i j}$ and $P_{i j}$ explicitly, without finding the limits as above.

Proposition 3.3. If the subspace spanned by $\mathscr{D}(A) \cup \mathscr{D}(B)$ is dense in $\mathscr{T}$, $A_{11}+B_{11}$ is injective. In this case, let $X$ be any one of $A_{11}-B_{11}, A_{21}, B_{21}$ and $Y$ any one of $A_{11}-B_{11}, A_{12}, B_{12}$, and suppose $X\left(A_{11}+B_{11}\right)^{-1}$ is closable. Then

$$
[X S Y]=-\left[X\left(A_{11}+B_{11}\right)^{-1}\right]^{-} Y .
$$

Proof. Suppose $\left(A_{11}+B_{11}\right) x=0$; then we must have $A_{11} x=B_{11} x=0$, and from Theorem 1.1(i), $A_{21} x=B_{21} x=0$ also. Thus

$$
\left\langle x, A_{11} y_{1}+A_{12} y_{2}+B_{11} y_{3}+B_{12} y_{4}\right\rangle=0 \quad \forall y_{1}, y_{2}, y_{3}, y_{4} .
$$

But $\left\{A_{11} y_{1}+A_{12} y_{2}+B_{11} y_{3}+B_{12} y_{4}\right\}=\operatorname{span} \mathscr{D}(A) \cup \mathscr{D}(B)$, so $x=0$.

In this case, we see that

$$
S_{n}=\left(A_{11}+B_{11}\right)^{-1}\left[\left(1-\frac{1}{2}\left(A_{11}+B_{11}\right)\right)^{n}-1\right], \quad n=0,1,2, \ldots,
$$

and since $A_{11}+B_{11}$ is injective, selfadjoint, and bounded by 2 ,

$$
\lim _{n \rightarrow \infty}\left[\left(1-\frac{1}{2}\left(A_{11}+B_{11}\right)\right)^{n}-1\right] x=-x \quad \forall x \in \mathcal{X} .
$$

Now for any $x \in \mathcal{H}$ we have

$$
X\left(A_{11}+B_{11}\right)^{-1}\left[\left(1-\frac{1}{2}\left(A_{11}+B_{11}\right)^{n}-1\right] Y x=X S_{n} Y x \rightarrow[X S Y] x\right.
$$

and

$$
\left[\left(1-\frac{1}{2}\left(A_{11}+B_{11}\right)\right)^{n}-1\right] Y x \rightarrow-Y x .
$$

Hence if $X\left(A_{11}+B_{11}\right)^{-1}$ is closable, then $Y x \in \mathscr{D}\left(\left[\left(A_{11}+B_{11}\right)^{-1}\right]^{-}\right)$and 


$$
\left[X\left(A_{11}+B_{11}\right)^{-1}\right]^{-} Y X=-[X S Y] x .
$$

Corollary 3.4. Let the span of $\mathscr{D}(A) \cup \mathscr{D}(B)$ be dense in $\mathcal{K}$, and suppose the domains

$$
\text { D }\left(\left(A_{11}+B_{11}\right)^{-1}\left(A_{11}-B_{11}\right)\right), \quad \mathscr{D}\left(\left(A_{11}+B_{11}\right)^{-1} A_{12}\right), \quad \mathscr{D}\left(\left(A_{11}+B_{11}\right)^{-1} B_{12}\right)
$$

are all dense in $\mathcal{K}_{0}$. Then

$$
\begin{aligned}
& S_{11}=S_{13}=S_{31}=S_{33}=\frac{1}{4}\left(A_{11}+B_{11}\right)-\frac{1}{4}\left[\left(A_{11}-B_{11}\right)\left(A_{11}+B_{11}\right)^{-1}\left(A_{11}-B_{11}\right)\right]^{-}, \\
& S_{12}=S_{32}=S_{21}^{*}=S_{23}^{*}=\frac{1}{2} A_{12}-\frac{1}{2}\left[\left(A_{11}-B_{11}\right)\left(A_{11}+B_{11}\right)^{-1} A_{12}\right]^{-}, \\
& S_{14}=S_{34}=S_{41}^{*}=S_{43}^{*}=\frac{1}{2} B_{12}+\frac{1}{2}\left[\left(A_{11}-B_{11}\right)\left(A_{11}+B_{11}\right)^{-1} B_{12}\right]^{-}, \\
& S_{24}=S_{42}^{*}=\left[A_{21}\left(A_{11}+B_{11}\right)^{-1} B_{12}\right]^{-} \\
& S_{22}=A_{22}-\left[A_{21}\left(A_{11}+B_{11}\right)^{-1} A_{12}\right]^{-} \\
& S_{44}=B_{22}-\left[B_{21}\left(A_{11}+B_{11}\right)^{-1} B_{12}\right]^{-} .
\end{aligned}
$$

Let $X$ be any one of $\left(A_{11}-B_{11}\right), A_{21}, B_{21}$, and suppose $x \in \mathscr{D}\left(\left(A_{11}\right.\right.$ $\left.\left.+B_{11}\right)^{-1} X^{*}\right), \quad y \in \mathbb{D}\left(\left(A_{11}+B_{11}\right)^{-1}\right)$. Then $\left\langle y,\left(A_{11}+B_{11}\right)^{-1} X^{*} x\right\rangle=\left\langle X\left(A_{11}\right.\right.$ $\left.\left.+B_{11}\right)^{-1} y, x\right\rangle$, showing $\left(\left(A_{11}+B_{11}\right)^{-1} X^{*}\right)^{*} \supset X\left(A_{11}+B_{11}\right)^{-1}$ and hence that $X\left(A_{11}+B_{11}\right)^{-1}$ is closable. The corollary follows from Proposition 3.2.

Analogous results can be obtained for the $P_{i j}$; we leave the exact statement of these results to the reader.

4. Sufficient conditions for $\mathscr{D}(A+B)$ to be dense. From the preceding section and Proposition 2.2, we have immediately

\section{Proposition 4.1.}

(i) $\mathscr{D}(A+B)$ is dense in $\mathcal{H}$ iff $A_{11}+B_{11}+\left[\left(A_{11}-B_{11}\right) S\left(A_{11}+B_{11}\right)\right]>0$.

(ii) $\mathcal{D}(B A)$ is dense in $\mathcal{T}$ iff $A_{11}+\left[A_{12} T A_{21}\right]>0$.

These conditions may be interpreted in the appropriate way if the conditions of Corollary 3.4 hold (or its analogue for products). It is interesting to rederive in this way known results in the cases when one of the operators $A$ or $B$ is bounded, or when $|A|$ and $|B|\left(\right.$ i.e. $\left(A^{*} A\right)^{1 / 2}$ and $\left.\left(B^{*} B\right)^{1 / 2}\right)$ commute. A somewhat less trivial result is given by the following theorem.

Theorem 4.2. Let $A$ and $B$ be closed linear operators, with dense domains, and suppose

$$
\operatorname{Re}\left(A_{11} x, B_{11} x\right)>0 \quad \forall x \in \mathscr{K}, x \neq 0 .
$$

Then $\mathscr{D}(A+B)$ is dense in 36

Proof. We have, for every nonzero $x \in \mathcal{H}$, 


$$
\begin{aligned}
\left\|\left(A_{11}+B_{11}\right) x\right\|^{2} & =\left(\left(A_{11}^{2}+B_{11}^{2}\right) x, x\right)+2 \operatorname{Re}\left(A_{11} x, B_{11} x\right) \\
& >\left(\left(A_{11}^{2}+B_{11}^{2}\right) x, x\right)-2 \operatorname{Re}\left(A_{11} x, B_{11} x\right) \\
& =\left\|\left(A_{11}-B_{11}\right) x\right\|^{2} .
\end{aligned}
$$

Thus for every $x \in R\left(A_{11}+B_{11}\right), x \neq 0$,

$$
\left\|\left(A_{11}-B_{11}\right)\left(A_{11}+B_{11}\right)^{-1} x\right\|<\|x\| .
$$

Thus $\left(A_{11}-B_{11}\right)\left(A_{11}+B_{11}\right)^{-1}$ is closable, and $\left(A_{11}+B_{11}\right)^{-1}\left(A_{11}-B_{11}\right)$ is defined on the whole space. For any $x \neq 0$, we have

$$
\left\|\left(A_{11}-B_{11}\right)\left(A_{11}+B_{11}\right)^{-1}\left(A_{11}-B_{11}\right) x\right\|<\left\|\left(A_{11}-B_{11}\right) x\right\|<\left\|\left(A_{11}+B_{11}\right) x\right\|,
$$

so by the triangle inequality,

$$
\begin{aligned}
4\left\|S_{11} x\right\| & =\left\|\left[\left(A_{11}+B_{11}\right)-\left(A_{11}-B_{11}\right)\left(A_{11}+B_{11}\right)^{-1}\left(A_{11}-B_{11}\right)\right] x\right\| \\
& \geqslant\|\|\left(A_{11}+B_{11}\right) x\|-\|\left(A_{11}-B_{11}\right)\left(A_{11}+B_{11}\right)^{-1}\left(A_{11}-B_{11}\right) x\|\|>0 .
\end{aligned}
$$

The result now follows from Proposition 2.2.

5. Characteristic matrices of sums and products. We use the results of $\$ 3$ to calculate the characteristic matrices of the sum and product of two closed linear operators.

Let $E_{4}$ be the projection operator in $\Im^{4}$ with matrix

$$
\left[\begin{array}{cccc}
1 & 0 & 0 & 0 \\
0 & \frac{1}{2} 1 & 0 & \frac{1}{2} 1 \\
0 & 0 & 1 & 0 \\
0 & \frac{1}{2} 1 & 0 & \frac{1}{2} 1
\end{array}\right], \quad 1 \in \mathscr{B}(\mathcal{C}) .
$$

Then $E_{4} S(A, B) \mathcal{H} C^{4}=\left\{\left(x, \frac{1}{2}(A+B) x, x, \frac{1}{2}(A+B) x\right) \mid x \in \mathscr{D}(A+B)\right\}$ and the projection onto the closure of the range of $E_{4} S(A, B)$ is easily seen to have the form

$$
\frac{1}{2}\left[\begin{array}{ll}
G\left(\frac{1}{2}(A+B)\right) & G\left(\frac{1}{2}(A+B)\right) \\
G\left(\frac{1}{2}(A+B)\right) & G\left(\frac{1}{2}(A+B)\right)
\end{array}\right], \quad G\left(\frac{1}{2}(A+B)\right) \in \mathscr{B}_{(}\left(\mathcal{F C}^{2}\right),
$$

where $G\left(\frac{1}{2}(A+B)\right)=$ characteristic matrix of $\frac{1}{2}(A+B)$. We can thus find $G\left(\frac{1}{2}(A+B)\right)$ by using the following result.

Lemma 5.1. If $C$ is any linear operator with $\|C\|=1$, the projection onto the closure of the range of $C$,

$$
R_{C}=\lim _{n \rightarrow \infty}\left(1-\left(1-C C^{*}\right)^{n}\right)
$$

in the strong operator topology. 
Proof. For any operator $T$, let $N_{T}$ be the projection onto the nullspace of $T$. Then we have $R_{C}=1-N_{C^{*}}=1-N_{C C^{*}}$. But $1=\|C\|^{2}=\left\|C C^{*}\right\| \geq C C^{*}$ $\geq 0$, so if

$$
C C^{*}=\int_{0}^{1} \lambda d E(\lambda)
$$

is the spectral decomposition of $C C^{*}$,

$$
\left(1-C C^{*}\right)^{n}=\int_{0}^{1}(1-\lambda)^{n} d E(\lambda) .
$$

Since $N_{C C^{*}}=E(\{0\})$, it follows that $N_{C C^{*}}=\lim _{n \rightarrow \infty}\left(1-C C^{*}\right)^{n}$ in the strong operator topology.

Theorem 5.2. Let $A$ and $B$ be any two closed linear operators, and $\left(A_{i j}\right),\left(B_{i j}\right)$ their characteristic matrices. Then the characteristic matrix of $\frac{1}{2}(A+B)$ is given by

$$
\begin{aligned}
& 1-G(1 / 2(A+B))=\lim _{n=\infty} \frac{1}{2^{n}}((1-G(A))+(1-G(B))
\end{aligned}
$$

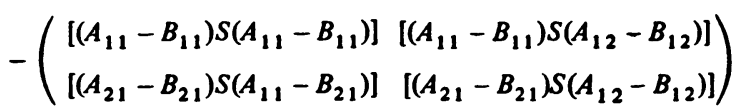

$$
\begin{aligned}
& =\lim _{n \rightarrow \infty}\left(1-\left(\begin{array}{cc}
2 S_{11} & S_{12}+S_{14} \\
S_{21}+S_{41} & 1 / 2\left(S_{22}+S_{24}+S_{42}+S_{44}\right)
\end{array}\right)\right)^{n}
\end{aligned}
$$

in the strong operator topology (where $\left[\left(A_{21}-B_{21}\right) S\left(A_{12}-B_{12}\right)\right]$ etc. are defined as in Theorem 3.1).

Proof. It is clear that it is sufficient to prove $1-G\left(\frac{1}{2}(A+B)\right)$ equal to the expression after the second equality sign above, and that terms such as $\left[\left(A_{21}-B_{21}\right) S\left(A_{12}-B_{12}\right)\right]$ exist. Letting $C=E_{4} S(A, B)$, we see $\|C\|=1$, unless $C=0$. The second case occurs when $G\left(\frac{1}{2}(A+B)\right)=0$, and the theorem is trivially true. Also,

$$
\begin{aligned}
C C^{*}= & E_{4} S(A, B) E_{4} \\
& =\frac{1}{2}\left[\begin{array}{cccc}
2 S_{11} & S_{12}+S_{14} & 2 S_{11} & S_{12}+S_{14} \\
S_{21}+S_{41} & T & S_{21}+S_{41} & T \\
2 S_{11} & S_{12}+S_{14} & 2 S_{11} & S_{12}+S_{14} \\
S_{21}+S_{41} & T & S_{21}+S_{41} & T
\end{array}\right]
\end{aligned}
$$

where $T=\frac{1}{2}\left(S_{22}+S_{24}+S_{42}+S_{44}\right)$.

The theorem then follows from the lemma above and the fact that if $x$ is an element of any ring with unit,

$$
1-\left(1-\left(\begin{array}{ll}
x & x \\
x & x
\end{array}\right)\right)^{n}=\frac{1}{2}\left(\begin{array}{ll}
1-(1-2 x)^{n} & 1-(1-2 x)^{n} \\
1-(1-2 x)^{n} & 1-(1-2 x)^{n}
\end{array}\right)
$$


We have found no simple "termwise" formula which would enable the characteristic matrix of the sum to be calculated as easily as the sum bicharacteristic matrix. Thus we have no simple formula which determines when the sum of two closed operators has a closure.

To obtain the characteristic matrix of the product is even easier than for the sum. We have

Theorem 5.3. Let $A$ and $B$ be any two closed linear operators, and $\left(A_{i j}\right),\left(B_{i j}\right)$ their characteristic matrices. Then the characteristic matrix of $B A$ is given by

$$
1-G(B A)=\lim _{n \rightarrow \infty}\left[1-\left[\begin{array}{ll}
P_{11} & P_{14} \\
P_{41} & P_{44}
\end{array}\right]\right]^{n}
$$

(where $P_{i, j}=i, j$ element of $P(A, B)$ ).

Proof. Let $E_{5}$ be the projection in $\mathcal{H}^{4}$ with matrix

$$
\left[\begin{array}{llll}
1 & 0 & 0 & 0 \\
0 & 0 & 0 & 0 \\
0 & 0 & 0 & 0 \\
0 & 0 & 0 & 1
\end{array}\right]
$$

Then $E_{5} P(A, B) \mathcal{C}^{4}=\{(x, 0,0, B A x) \mid x \in \mathscr{D}(B A)\}$ and the projection onto the closure of this subspace has the form

$$
\left[\begin{array}{cccc}
G(B A)_{11} & 0 & 0 & G(B A)_{12} \\
0 & 0 & 0 & 0 \\
0 & 0 & 0 & 0 \\
G(B A)_{21} & 0 & 0 & G(B A)_{22}
\end{array}\right]
$$

The theorem now follows from Lemma 5.1.

For the case of the product of two operators, we have obtained an expression for the limit, in terms of known operators. The expression has the form

$$
\begin{aligned}
& G(B A)=\left[\begin{array}{ll}
P_{11} & P_{14} \\
P_{41} & P_{44}
\end{array}\right] \\
& +2\left[\begin{array}{l}
{\left[A_{12}\left(A_{22}+B_{11}\right)^{-1} B_{11}\left(1-2 P_{22}\right)^{-1} B_{11}\left(A_{22}+B_{11}\right)^{-1} A_{21}\right]^{-}} \\
{\left[B_{21}\left(A_{22}+B_{11}\right)^{-1} A_{22}\left(1-2 P_{22}\right)^{-1} B_{11}\left(A_{22}+B_{11}\right)^{-1} A_{21}\right]^{-}}
\end{array}\right. \\
& \left.\left[A_{12}\left(A_{22}+B_{11}\right)^{-1} B_{11}\left(1-2 P_{22}\right)^{-1} A_{22}\left(A_{22}+B_{11}\right)^{-1} B_{12}\right]^{-}\right] \\
& \left.\left[B_{21}\left(A_{22}+B_{11}\right)^{-1} A_{22}\left(1-2 P_{22}\right)^{-1} A_{22}\left(A_{22}+B_{11}\right)^{-1} B_{12}\right]^{-}\right]
\end{aligned}
$$

in the case when all inverses exist, and the closures exist as bounded operators. An expression holding under more general conditions may be found, but we see little point in phrasing it. The expression above is too complicated to be of much use, and we shall not prove it here. One can use it to obtain a condition which determines when the product of two closed operators has a closure. 
6. Direct integrals of sums and products. In this section we apply the results of the preceding sections to the direct integral theory of closed operators. We use Dixmier [1] and Nussbaum [2] as basic references.

Let $Z$ be a Borel space, $\mu$ a positive measure on $Z, t \rightarrow \mathcal{H}(t)$ a $\mu$-measurable field of Hilbert spaces, $\mathcal{K}=\int_{Z}^{\oplus} \mathcal{H}(t) d \mu(t)$. Let $\mathcal{Q}$ be the abelian von Neumann algebra of diagonalisable operators. For each $t \in Z$, let $A(t)$ be a closed linear operator in $\mathcal{Y C}(t)$. We say that $t \rightarrow A(t)$ is a measurable field of closed operators iff $t \rightarrow A(t)_{i j}$ is a measurable field of bounded operators for each $i, j=1,2$. In this case we can define

$$
A_{i j}=\int_{z}^{\oplus} A(t)_{i j} d \mu(t),
$$

and it follows that the $A_{i j}$ satisfy Theorem 1.1 (i)-(iii). We define $A=\int_{\mathbf{Z}}^{\oplus} A(t) d \mu(t)$ to be the closed linear operator such that $G(A)=\left(A_{i j}\right)$.

Theorem 6.1 (Pallu de Barrière-Nussbaum). Let $A \eta Q^{\prime}$, i.e. $U A=A U$ for every unitary operator $U$ in $\&$. Then there exists a measurable field of closed operators, $t \rightarrow A(t)$, such that

$$
A=\int_{z}^{\oplus} A(t) d \mu(t)
$$

Furthermore

(i) $\mathscr{D}(A)=\{x \in \mathscr{C} \mid x(t) \in \mathscr{D}(A(t))$ almost everywhere (a.e.) and $t \rightarrow A(t)$ $x(t)$ is square-integrable $\}$.

(ii) If $x \in \mathscr{D}(A), t \rightarrow A(t) x(t)$ is measurable.

(iii) $\mathscr{D}(A)$ is dense iff $\mathscr{D}(A(t))$ is dense for almost all $t$. In this case, $t \rightarrow A(t)^{*}$ is measurable and $A^{*}=\int_{Z}^{\oplus} A(t)^{*} d \mu(t)$.

If $A$ is a closed linear operator and $A \eta \mathbb{Q}^{\prime}$, we say that $A$ is decomposable.

If $A$ and $B$ are bounded decomposable operators,

$$
\begin{aligned}
A+B & =\int_{Z}^{\oplus}(A(t)+B(t)) d \mu(t), \\
B A & =\int_{Z}^{\oplus} B(t) A(t) d \mu(t) .
\end{aligned}
$$

Nussbaum has proved that if $A$ and $B$ are decomposable, and each $A(t)$ and $B(t)$ is bounded, then $A+B$ and $B A$ are closable and the above equations hold with $A+B, B A$ replaced by $[A+B]^{-},[B A]^{-}$respectively. We now extend this result to the case where $A$ and $B$ are arbitrary decomposable operators.

Theorem 6.2. Let $A$ and $B$ be closed decomposable linear operators. Then

(i) $\mathscr{D}(A+B)$ is dense iff $\mathscr{D}(A(t)) \cap \mathscr{D}(B(t))$ is dense in $\mathcal{G}(t)$ for almost all $t$.

(ii) $A+B$ is closable iff $A(t)+B(t)$ is closable for almost all $t$. In this case, $t \rightarrow[A(t)+B(t)]^{-}$is measurable and 


$$
[A+B]^{-}=\int_{Z}^{\bullet}[A(t)+B(t)]^{-} d \mu(t) .
$$

Proof. Let $\left(A_{i j}\right),\left(B_{i j}\right)$ be the characteristic matrices of $A$ and $B$. Then by definition,

$$
A_{i j}(t)=(A(t))_{i j} \quad \text { a.e., } \quad B_{i j}(t)=(B(t))_{i j} \quad \text { a.e. }
$$

Let $S_{n}$ be the operator defined in Theorem 3.1. Then since $S_{n}$ is a polynomial in the bounded decomposable operators $A_{i j}, B_{i j}, S_{n}$ is decomposable, and furthermore

$$
S_{n}(t)=-\frac{1}{2} \sum_{i=1}^{n}\left(\begin{array}{c}
n \\
i
\end{array}\right)\left[-\frac{1}{2}\left(A_{11}(t)+B_{11}(t)\right)\right]^{i-1} \quad \text { a.e. }
$$

If $X$ is any one of $A_{11}-B_{11}, A_{21}, B_{21}$, and $Y$ any one of $A_{11}-B_{11}, A_{12}, B_{12}$, then $X S_{n} Y$ is decomposable and

$$
\left(X S_{n} Y\right)(t)=X(t) S_{n}(t) Y(t) \text { a.e. }
$$

By Theorem 3.1, $\left\{X S_{n} Y\right\}$ and $\left\{X(t) S_{n}(t) Y(t)\right\}$ converge in the strong operator topology, and since $X S_{n} Y \rightarrow[X S Y]$, there is a subsequence $\left\{n_{i}\right\}$ such that

$$
\left(X S_{n_{i}} Y\right)(t) \rightarrow[X S Y](t) \text { a.e. }
$$

Hence, denoting the limit of $\left\{X(t) S_{n}(t) Y(t)\right\}$ by $[X(t) S Y(t)]$, we have

$$
[X(t) S Y(t)]=[X S Y](t) \text { a.e. }
$$

It follows that $S(A, B)_{i j}$ is decomposable and for $i, j=1, \ldots, 4$,

$$
S(A, B)_{i j}(t)=S(A(t), B(t))_{i j} \text { a.e. }
$$

Now using Theorem 5.2, the same argument may be applied again to obtain $G\left(\frac{1}{2}(A+B)\right)$ is decomposable and

$$
G\left(\frac{1}{2}(A+B)\right)(t)=G\left(\frac{1}{2}(A(t)+B(t))\right) \text { a.e. }
$$

The theorem now follows immediately from Theorems 1.1 and 1.2 and the fact that a bounded decomposable operator $C$ is injective iff $C(t)$ is injective for almost all $t$.

Minor modifications of the above proof give us

Theorem 6.3. Let $A$ and $B e$ be closed decomposable linear operators. Then

(i) $\mathscr{D}(B A)$ is dense iff $\mathscr{D}(B(t) A(t))$ is dense in $\Im(t)$ for almost all $t$.

(ii) $B A$ is closable iff $B(t) A(t)$ is closable for almost all $t$. In this case $t \rightarrow[B(t) A(t)]^{-}$is measurable and

$$
[B A]^{-}=\int_{z}^{\oplus}[B(t) A(t)]^{-} d \mu(t) .
$$




\section{REFERENCES}

1. J. Dixmier, Les algèbres d'opérateurs dans l'espace Hilbertien (Algèbres de von Neumann), Gauthier-Villars, Paris, 1969.

2. A. E. Nussbaum, Reduction theory for unbounded closed operators in Hilbert space, Duke Math. J. 31 (1964), 33-44. MR 28 \#2438.

3. R. Pallu de Barrière, Décomposition des opérateurs non bornées dans les sommes continues d'espace de Hilbert, C. R. Acad. Sci. Paris Sér. A 232 (1951), 2071-2073. MR 12, 837.

4. M. H. Stone, On unbounded operators in Hilbert space, J. Indian Math. Soc. 15 (1951), 155-192. MR 14, 565.

5. J. von Neuman, Functional operators. II. The geometry of orthogonal spaces, Ann. of Math. Studies, no. 22, Princeton Univ. Press, Princeton, N. J., 1950. MR 11, 599.

Department of Mathematics, University of Auckiand, Auckland, New Zealand 